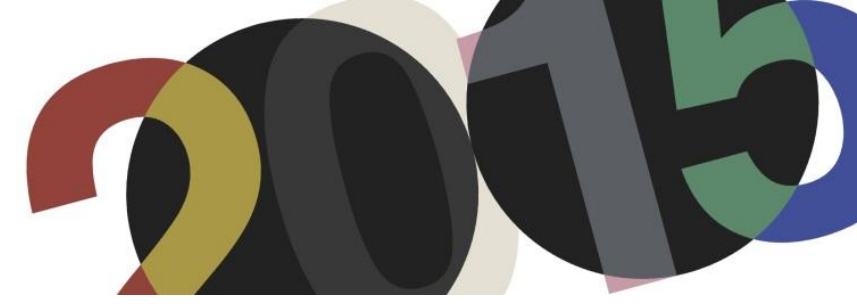

DOI: http://dx.doi.org/10.4995/LC2015.2015.1042

\title{
Une correspondance architecturale: Ionel Schein "enfant" de Le Corbusier
}

\author{
S. Berselli \\ Istituto di storia e teoria dell'arte e dell'architettura (ISA) \\ Accademia di architettura di Mendrisio - Università della Svizzera Italiana (USI)
}

\begin{abstract}
Résumé: Pour la génération d'architectes, tels Ionel Schein, qui se forment à Paris après la guerre, Le Corbusier représente le seul antidote à l'académisme "bozartien". La filiation corbuséenne se fait d'abord en clandestinité, à travers les livres et les projets, puis avec le contact direct ou par lettre, et représente un bassin inépuisable pour l'éducation permanente des jeunes à l'architecture. Les rencontres et la correspondance, ponctuée de divertissements intellectuels, permettent à Schein d'absorber le lexique corbuséen comme une nouvelle langue maternelle, jusqu'à la formation de son propre vocabulaire d'architecture. A la mort du maître, contré pendant toute sa vie par les institutions, Schein est dégoûté par la suite grotesque d'honneurs posthumes mise en scène : sa fidélité au message de Le Corbusier survit aux apparences et dans ses obituaires sans couronnes de lauriers on trouve le sens profond d'un héritage tant discuté et imposant.
\end{abstract}

Resumen: Para la generación de arquitectos que, como Ionel Schein, se forman en París después de la guerra, Le Corbusier es el único antídoto contra el academicismo de la Escuela de Bellas Artes. La filiación corbuseana se hace primero clandestinamente, a través de los libros y de los proyectos, y en seguida con el contacto directo o por carta, y representa una reserva inagotable por la educación de los jóvenes a la arquitectura. Las reuniones y la correspondencia, punteada de entretenimientos intelectuales, permiten a Schein de absorber el léxico corbuseano como una nueva lengua materna, hasta la formación de su propio vocabulario arquitectónico. A la muerte del maestro, Schein crítica duramente la serie grotesca de honores póstumos enscenada por las instituciones que lo obstaculizaron por toda su vida. Su fidelidad al mensaje de Le Corbusier sobrevive a las apariencias y en sus obituarios sin coronas de laurel se encuentra el significado más profundo de un legado tan discutido e imponente.

Mots clés: Ionel Schein; Claude Parent; Atelier 35 rue de Sèvres; Scuola CIAM Venezia 1952; CIAM IX 1953; correspondance.

Palabras clave: Ionel Schein; Claude Parent; Atelier 35 rue de Sèvres; Escuela CIAM Venecia 1952; CIAM IX 1953; correspondencia.

\section{Introduction \\ Objectifs, méthode, résultats}

Des nombreux écrits critiques que Ionel Schein (1927-2004) a dédié à Le Corbusier ${ }^{1}$ descends une filiation que jusqu'aujourd'hui a été peu étudiée, et seulement du côté du maître. Pendant mes recherches doctorales, dirigées par Prof. Carlo Olmo dès 2007, j'ai analysé l'œuvre d'Ionel Schein, architecturale et écrite. Cet étude dans un archive inexploré, intégré par la visite aux bâtiments réalisés et les entretiens aux protagonistes, a permis d'identifier des thématiques constantes dans la pensée et la production des deux non-architectes. Ma thèse doctorale vient de paraitre en italien, avec le titre Ionel Schein. Dall'habitat evolutivo all'architecture populaire ${ }^{2}$.

\footnotetext{
${ }^{1}$ Voir la Bibliographie, au point 6.

${ }^{2}$ Berselli, Silvia: Ionel Schein. Dall'habitat evolutivo all'architecture populaire. Mendrisio: Mendrisio Academy Press Cinisello Balsamo: Silvana Editoriale, 2015.
} 
Ma proposition pour le Congrès LC2015 a comme objectif la reconstruction d'une filiation qui commence avec la lecture des livres du maître et le pèlerinage de visite à ses bâtiments, se concrétise en 1952, quand Ionel Schein est chargé d'accueillir Le Corbusier à Venise, et termine seulement avec la mort de ce dernier. Le fonds Schein conservé au Frac Centre d'Orléans et l'archive de la Fondation Le Corbusier à Paris présentent plusieurs documents inédits ${ }^{3}$ qui témoignent l'intensité de la correspondance entre les deux : envois de textes polémiques ou programmatiques, commentaires sur chantiers et projets récents ou du passé, réflexions, congratulations pour chaque succès ou un petit mot de soutien pour les échecs. Tout ça, bien sûr, de la part de Schein ; mais il reçoit toujours une réponse positive, soit qu'il nécessite quelques dessins pour une publication ou bien pour une exposition, soit qu'il demande la production d'un texte original à donner aux jeunes étudiants ou la présence à une signature de livres.

L'étude de cette relation permet de mieux comprendre l'influence de Le Corbusier sur la génération des jeunes architectes qui bâtissent pendant la reconstruction, après la promulgation du Plan Courant. Déçus par l'Académie des Beaux-Arts, les institutions, les CIAM, ils trouvent une nouvelle énergie vitale dans l'atelier de Le Corbusier, qui ne leur empêche pas de choisir, après, de différents chemins, en devenant par exemple architectes radicales (Guy Rottier), engagés politiquement (Ionel Schein) ou plasticiens (Claude Parent). Les journaux de commentaires personnels que Schein écrit à Venise et à Aix-en-Provence représentent une critique ponctuelle, motivée et anti-héroïque de cette saison de renouvellement des CIAM, vue de l'intérieur. Le stage à l'atelier de la rue de Sèvres en 1953 permet à Schein de travailler aux dessins de l'Unité de Nantes-Rezé et de concentrer son attention sur le thème privilégié de l'architecture pour le plus grand nombre, sujet central du débat de l'après-guerre et de la riche production architecturale bâtie par Schein.

\section{Nous étions des clandestins \\ La leçon corbuséenne comme antidote à l'académisme "bozartien"}

Après avoir quitté la Roumanie en 1948, Ionel Schein déménage avec sa famille à Paris, où il s'inscrit à l'Ecole Nationale Supérieure des Beaux-Arts pour poursuivre ses études d'architecture, commencés après la guerre à l'Université de Bucarest. Pour comprendre le climat de l'Ecole, on peut se référer à l'autobiographie de Claude Parent, compagnon d'étude et de travail d'Ionel Schein du 1949 au 1955 : "En ce temps-là, déjà, l'école ne vivait plus que sur un faux prestige et des traditions défuntes. Divisée en atelier dirigés chacun par un «patron» choisi par les élèves, elle entretenait un climat de compétition artificielle entre les étudiants. D'atelier à atelier la lutte était féroce. Tous les coups étaient bons pour obtenir, dans chaque "écurie" pourrait-on dire, le plus possible de récompenses scolaires destinées non pas à préparer les jeunes à l'exercice correct de leur future profession, mais à tresser des couronnes de laurier aux architectes patrons les plus débrouillards, et les plus forts en gueule au jury, en réalité les plus compromis et pistonnés, complices volontaires de la léthargie d'une administration croupissante et veule" ${ }^{\prime 4}$. Comme en témoigne Claude Parent, les années Quarante et Cinquante montrent une phase de déclin graduel de l'Ecole ${ }^{5}$ et les nombreux projets de réforme de l'institution sont contestés et jamais appliqués ${ }^{6}$.

\footnotetext{
${ }^{3}$ Je remercie les archives suivantes : Fondation Le Corbusier, Paris (dorénavant abrégée FLC) ; Fonds Schein, FRAC Centre, Orléans (abrégé FRAC) ; Archive CIAM, GTA Archives, Zurich (abrégé GTA).

${ }^{4}$ Parent, Claude: Claude Parent Architecte. Paris: Laffont, 1975, pp. 17-18.

${ }^{5}$ Epron, Jean-Pierre (édité par): Architecture: une anthologie. Tome 2: les architectes et le projet. Liège: Pierre Mardaga, 1992, p. 135.

${ }^{6}$ Seulement en 1962 la réforme Querrien a été approuvée, mais le projet de loi est tardif, n'entre pas en vigueur et ne peut pas arrêter la dissolution des ateliers et la capitulation de l'institution avec les manifestations étudiantes de mai 1968.
} 
Au début des années Cinquante, André Gutton appelle Le Corbusier "le grand publiciste", le nom de ce "monsieur", considéré comme "le diable soi-même fait non-architecte”, , est interdit par l'Ecole et, si jamais il est prononcé, cela provoque des rires moqueurs parmi les étudiants et les professeurs ${ }^{9}$. Pendant cette période, la figure de Le Corbusier détermine la limite entre orthodoxie et révolution au sein de l'Ecole et son œuvre interdite est un liant exceptionnel, capable de cimenter un partenariat fructueux entre les jeunes rebelles, fatigués de respirer la poussière des institutions (Figures 1 et 2).
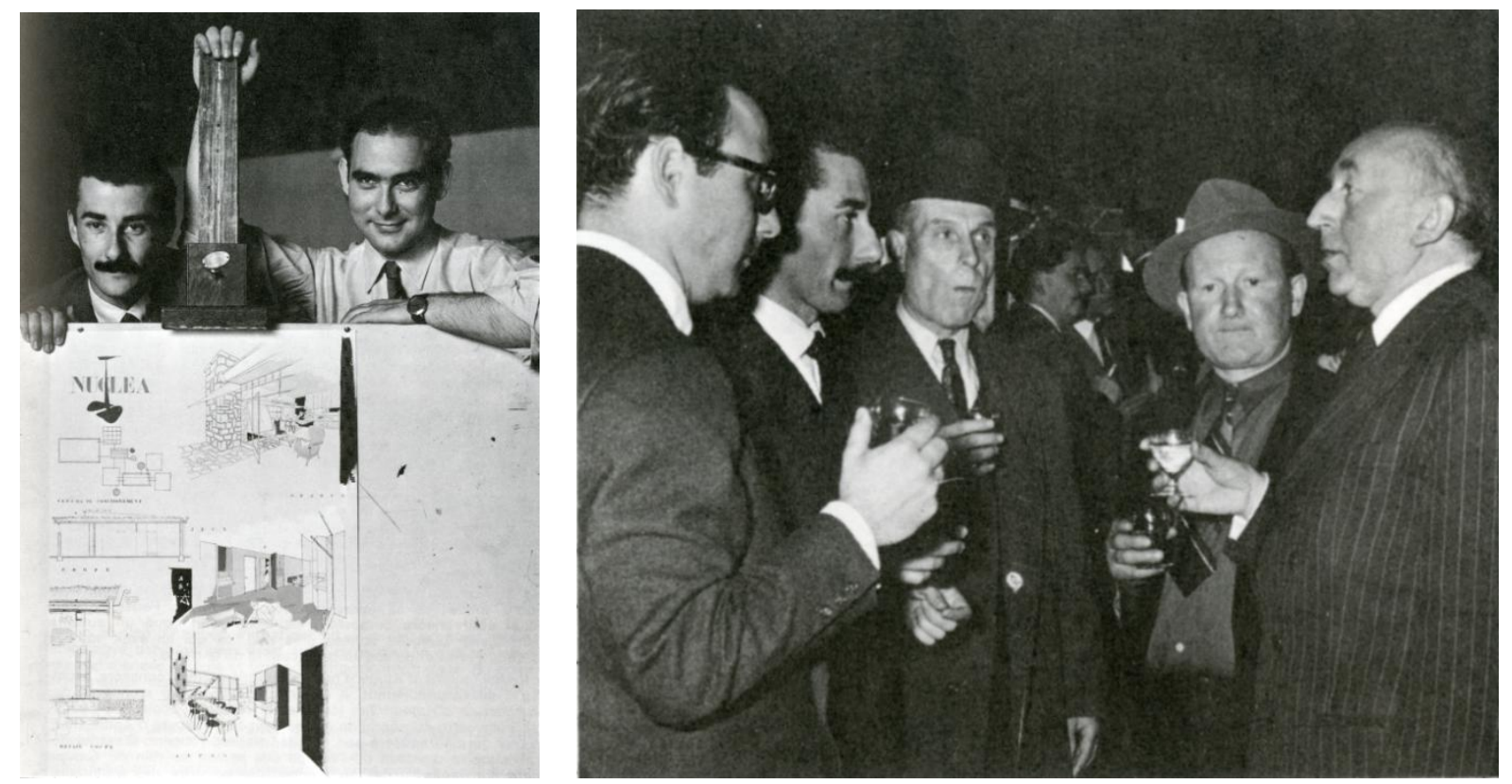

1. Claude Parent (à gauche) et Ionel Schein (à droite) avec le projet gagnant pour le concours «La Maison Française», 1952 (dans Ragon, Michel: Claude Parent. Monographie critique d'un architecte. Paris: Dunod, 1982, p. 33).

2. Concours national "La Maison Française", 1952. De gauche à droite : Ionel Schein, Claude Parent, les deux directeurs de l'ENSBA et Pierre Sonrel (ibidem, p. 32).

Ionel Schein et Claude Parent s'opposent à l'enseignement académique en produisant des textes polémiques adressés $A$ nos ainés...les jeunes ${ }^{10}$. La rébellion les unit de plus en plus, au point que Nicolas Schöffer se souvient d'eux "comme des jumeaux"111; cette complicité les fortifie jusqu'à trouver le courage de citer publiquement, et pour la première fois dans l'histoire de l'Ecole, Le Corbusier : "Sur un projet nous avions mis une citation de Le Corbusier, avec son nom. C'était la première fois que le nom de Le Corbusier apparaissait dans les ateliers. Le scandale a été énorme. Gutton a dit en amphi : "Parmi vous il y a un provocateur mal élevé». Mais plus tard nous avons eu de très bons échanges avec Gutton, qui nous a fait admettre à l'Ordre,

\footnotetext{
${ }^{7}$ Cfr. Ragon, Michel: Monographie critique d'un architecte: Claude Parent. Paris: Dunod, 1982, p. 27.

8 "...j'avais lu, dès quinze ans, les livres de Le Corbusier, où je me régalais des formes utiles d'avions et de bateaux, et accessoirement de quelques habitations et immeubles jetés dans la verdure. Mais il était interdit de parler alors de ce "monsieur » à l'école, car on le considérait comme le diable soi-même fait non-architecte", dans Parent, Claude 1975, p. 19.

9 "Non seulement l'enseignement qui lui est donné quai Malaquais ne ressemble en rien à Vers une architecture, mais le nom seul de Le Corbusier provoque alors la plus franche rigolade aussi bien parmi les élèves que parmi les professeurs", dans Ragon, Michel 1982, p. 13.

${ }^{10}$ Parent, Claude; Schein, Ionel : A nos ainés...les jeunes, document inédit daté 25 février 1953. FRAC.

11 "J'ai rencontré Claude Parent grâce à André Bloc et au Groupe Espace. Parent et Schein, qui étaient alors comme des jumeaux, m'ont invité dans leur atelier du boulevard Suchet. Ils étaient très jeunes, très sympathiques, deux jeunes loups qui rouaient dans les brancards". Entretien avec Nicholas Schöffer, juin 1980, dans Ragon, Michel 1982, p. 127.
} 
Parent et $\mathrm{moi}^{\prime \prime 12}$. En 1966, c'est bien Gutton qui favorise l'admission de Schein et Parent à l'Ordre des Architectes sur présentation d'un “dossier d'œuvres”. Il s'agit d'un procédé exceptionnel et rare utilisé en précédence pour l'admission de professionnels non diplômés, mais reconnus pour la qualité des œuvres, notamment Auguste Perret et Le Corbusier ${ }^{13}$.

Les années de la collaboration représentent pour Schein et Parent la période de formation à l'architecture, un processus d'apprentissage qui se déroule simultanément sur de différents niveaux, tout d'abord avec la critique de l'enseignement traditionnel, puis avec la fréquentation des protagonistes de l'avant-garde artistique. Dans cette période, ils produisent des projets résidentiels cohérents avec les prescriptions du Ministère de la Reconstruction et de l'Urbanisme (MRU) et ils publient leurs conceptions au même temps sur les pages des revues spécialisées et dans les hebdomadaires populaires, comme "Elle" et "La Maison Française"14. La nature de cette collaboration a été reconstruite par Schein dans le texte Nous étions des clandestins : "A l'Ecole des Beaux-Arts nous étions des clandestins; les deux seuls ou les deux rares à avoir manifesté notre marginalité, notre opposition au système "bozartien". Nous avons refusé de faire le diplôme, c'est-à-dire de reconnaître le système, alors que nous avions les valeurs et le temps pour le faire. Nous sommes restés une dizaine d'années aux Beaux-Arts, tout en entrant dans la profession par effraction"15.

\section{La main du maître forçant la nôtre}

\section{La filiation corbuséenne comme éducation permanente à l’architecture}

Dans le vaste réseau de relations professionnelles tissé par Ionel Schein, se démarque par sa continuité chronologique et son intensité le lien établi avec Le Corbusier. Le jeune Schein manifeste très tôt son admiration pour les écrits et les œuvres de Le Corbusier, et peut-être encore plus pour son charisme en tant que professionnel pas diplômé, contré par les institutions académiques et au même temps adoré par les avant-gardes. En ces quelques mots, Claude Parent reconstruit la filiation corbuséenne de sa formation, et de celle d'Ionel Schein, à l'architecture : "Enfant de Le Corbusier je l'ai été, je vous l'ai dit, avant même de savoir ce qu'était l'architecture. Enfants de Le Corbusier, nous les fumes, Ionel Schein et moi, quand nous avons construit nos trois premières maisons après celle du concours. Les maisons Morpain, Le Jeannic, Hardy, petites cabanes de béton, sont venues au monde avec la main du maître forçant la nôtre ${ }^{\prime 16}$.

Le premier contact avec l'Atelier de la rue de Sèvres se fait par une lettre qu'Ionel Schein et Claude Parent écrivent aux délégués nationaux des CIAM ${ }^{17}$. La lettre, datée du 7 janvier $1952^{18}$, exhorte les délégués des CIAM à coordonner les efforts des étudiants des écoles d'architecture et d'urbanisme pour créer dans chaque

\footnotetext{
${ }^{12}$ Schein, Ionel: Nous étions des clandestins, dans Ragon, Michel 1982, p. 190.

13 "...en 1966, selon une enquête du Monde, avait obtenu le privilège de figurer parmi le huit professionnels inscrits à l'Ordre sans posséder de diplôme...", dans Ragon, Michel 1982, p. 18. Claude Parent indique l'année 1974 comme date de l'enquête, dans Parent, Claude 1975, p. 60.

${ }^{14}$ Cfr. par exemple : Schein, Ionel; Parent, Claude: Maison Française 1953. La Maison Française. № 69, juillet 1953, pp. 3 14. Raimond, Anne-Marie: Un toit pour chaque français (1 et 2). Elle. $\mathrm{N}^{\circ} 399,27$ juillet 1953, pp. 24-29; Elle. $\mathrm{N}^{\circ}$ 400, 3 aout 1953, pp. 26-29. Schein, Ionel; Parent, Claude: Essai pour un habitat individuel évolutif et Habitation à Ville d'Avray. L'Architecture d'Aujourd'hui. N 49, octobre 1953, pp. 4-5 et pp. 10-13.

${ }^{15}$ Schein, Ionel: Nous étions des clandestins. Ragon, Michel 1982, pp. 190-192.

${ }^{16}$ Parent, Claude 1975, p. 28.

${ }^{17}$ Ils déclarent avoir trouvé les adresses dans les dernières pages du livre : Giedion, Siegfried: A Decade of New Architecture - Dix ans d'Architecture Contemporaine. Zurich: Girsberger, 1951, pp. 231-232.

${ }^{18}$ La copie envoyée à Le Corbusier est à la FLC, D2-20-333 ; celle de Siegfried Giedion est au GTA, 42-SG-40-509. Les deux lettres sont identiques.
} 
pays un groupe de jeunes. Ils exigent une investiture officielle, de façon que ces représentants des étudiants puissent collaborer avec les membres du Congrès et transmettre leur message dans les universités.

La lettre reçoit plusieurs réponses, de la part de Candilis, Emery, Giedion ${ }^{19}$, Lods, Neutra, Roth, Sert, Le Corbusier e Wogenscky ${ }^{20}$, comme spécifié par Schein et Parent en juin 1952 dans Présences ${ }^{21}$. Ce texte fait partie des Documents de Sigtuna 1952 et renouvelle l'appel aux délégués CIAM fait par lettre. Les auteurs demandent une investiture officielle des groupes des jeunes, mais surtout ils exigent une prise de position, de la part des membres des CIAM, au sujet de la formation en architecture. Dans ses textes, et encore dans ses dernières lettres à Le Corbusier, Schein dénonce à plusieurs reprises le problème de l'enseignement de l'architecture en France et menace le fantôme de la chute dans un "nouvel académisme", au point que ses mots semblent parfois prévoir les événements du Mai '68.

En cherchant une alternative à l'Académie, Ionel Schein participe à l'école d'été CIAM, tenue du 10 Septembre au 9 Octobre 1952 à l'IUAV de Venise, sous la direction de Franco Albini, Ignazio Gardella, Ernesto Nathan Rogers et Giuseppe Samonà. Pour mieux garder le souvenir de cette expérience, il écrit, pendant toute la durée de l'école, un journal qui représente un témoignage exceptionnel du climat tendu, parfois asphyxiante, des débats menés dans la ville lagunaire. Entre conférences répétitives et balades trop touristiques, Schein se montre critique et ennuyé, ne pouvant plus supporter les contraintes de la "mise en grille", jusqu'à se demander : "Quo vadis CIAM ?"22. Pendant cette période, Venise accueille aussi le Congrès Unesco et la Conférence internationale des Artistes $^{23}$, dont Le Corbusier est l'un des créateurs. Le jour 21 Septembre, Schein est chargé d'accueillir Le Corbusier à la gare de Venise et il note dans son journal : "Dimanche. Repos. Réception ridicule de L.C. à la gare, comme de petits enfants... marche à pied avec lui à travers Venise. Remarques ridicules et artificieuses $^{\prime 24}$. Deux jours plus tard, Le Corbusier donne une conférence que Schein considère entre les expériences les plus importantes de l'école d'été : "deux heures, mais des choses nouvelles et intéressantes : les Indes surtout" ${ }^{\prime 25}$. Selon Schein, le nouvel "organicisme" de Le Corbusier, descends de la "destruction de la machine par la machine" et porte à la construction d'un nouveau "microcosme",26.

Déçu par l'école CIAM et par ses pairs, Schein se rapproche progressivement de la figure qui constitue dans cette période de formation sa référence principale : Le Corbusier. Avec Parent, il fait un stage à l'Atelier de la rue de Sèvres en 1953, on ne peut pas déterminer si avant ou après le CIAM IX. À la Fondation Le Corbusier ils n'existent pas de traces du passage des jeunes à l'Atelier, tandis que le FRAC d'Orléans conserve une note de payement pour le "dessinateur" Ionel Schein ${ }^{27}$. Claude Parent raconte que ce stage a été bref, quelques mois, et consacré à la production de dessins pour le rez-de-chaussée de l'Unité d'Habitation de Nantes-Rézé, sous la

\footnotetext{
${ }^{19}$ Lettre de Siegfried Giedion à Claude Parent et Ionel Schein du 19 janvier 1952. GTA, 42-SG-40-508.

${ }^{20}$ Lettre d'André Wogenscky à Claude Parent et Ionel Schein du 14 janvier 1952. FLC, D2-20-334.

${ }^{21}$ Schein, Ionel; Parent, Claude: Présences, 23 juin 1952, dans Les documents de Sigtuna 1952, reçu par A. Roth le jour 20 aout 1952. GTA.

22 «30/9 Mise en grille... ceci veut dire... je me le demande. Enfin, puisqu'ils l'ont demandé soyons des élèves consciencieux... Quo vadis CIAM?» dans Schein, Ionel: Journal de l'Ecole d'été CIAM, manuscrit, 1952. FRAC.

${ }^{23}$ Les documents sont au FRAC et à la FLC, U3-7, 317-321.

${ }^{24}$ Schein, Ionel: Journal de l'Ecole d'été CIAM, manuscrit, 1952. FRAC.

${ }^{25}$ Ibidem.

${ }^{26}$ Ibidem.

27 "Atelier Le Corbusier, 29 jan 1954. Salaires et Rétributions payés pendant l'année 1953. Monsieur SCHEIN Yonel, Dessinateur. 22, Av. De Versailles - Paris $\left(X V I^{\circ}\right)$. Soixante-quatorze mille trois cent vingt francs (74.320 Frs.) Somme que nous avons déclarée aux Contributions Directes au titre de vos revenus pour l'année 1953". FRAC.
} 
direction de Iannis Xenakis. Le caractère impossible de ce dernier aurait été la cause du départ de Schein, suivi un mois plus tard par Claude Parent ${ }^{28}$.

En peu de temps après la première lettre aux délégués CIAM, les deux étudiants ont gagné considération auprès d'André Wogenscky, qui charge Claude Parent de se rendre à Aix-en-Provence pour l'organisation du CIAM $\mathrm{IX}^{29}$. Le Groupe CIAM-Jeunes se constitue à Paris ${ }^{30}$, sous la direction de Wogenscky et avec la collaboration de Georges Candilis ; la question à l'ordre du jour est l'habitat et les deux jeunes sont bientôt impliqués dans un débat auquel vont participer les protagonistes de la scène architecturale internationale ${ }^{31}$.

Le CIAM IX d'Aix-en-Provence se pose comme objectif la définition d'une Charte de l'Habitat, qui puisse jouer en architecture le rôle détenu par la Charte d'Athènes en urbanisme. Ce document univoque ne verra jamais le jour, en raison des différences de pensée irréconciliables au sein du Congrès. Le CIAM d'Aix marque une fracture $^{32}$ et registre la montée d'une nouvelle génération (les Smithson, Candilis et Bakema), qui remet en question le fonctionnalisme de la Charte d'Athènes.

Ionel Schein participe au Congrès et publie son compte-rendu sur «L'Architecture d'Aujourd'hui», juste après celui du directeur de la revue, André Bloc ${ }^{33}$. Dans son article, le jeune architecte d'origine roumaine utilise un lexique spécifique, appris au Congrès et bientôt assimilé, avec mots et locutions comme habitat, logis, taudis, prolongements du logis, le plus grand nombre, cadre de vie, aménagement du territoire. Pendant toute sa vie, Schein utilise ce vocabulaire, qui oriente sa réflexion vers la définition du thème centrale de sa production, l'architecture populaire ${ }^{34}$, sa version personnelle de la Charte de l'Habitat. Le CIAM IX instille dans le jeune architecte la graine de la réflexion sur le logement social, un sujet qui ensuite l'anime pendant toute sa production. Son premier manifeste pour un habitat individuel évolutif ${ }^{35}$ représente un trait-d'union linguistique avec les CIAM et germine parmi les papiers de notes du Congrès d'Aix-en-Provence : "Fonction habiter : varie suivant l'évolution de la société. Ce que l'homme construit pour habiter doit pouvoir perpétuellement $\underline{\text { changer }}^{\prime \prime 36}$. Cette prise de conscience amène Schein à abandonner les plans-types en vogue pour repenser le problème de la résidence avec un point de vue plus large, soit avec un plan d'“aménagement du territoire" dans lequel les "prolongements du logis" permettent le développement de la vie des hommes à l'intérieur et à l'extérieur du logement.

\footnotetext{
${ }^{28}$ Entretien avec Claude Parent recueilli par Silvia Berselli en novembre 2008.

${ }^{29}$ Lettre d'André Wogenscky et R. Andreini à Claude Parent du 12 décembre 1952. Wogenscky et Andreini envoient à Claude Parent un chèque pour le remboursement des frais de voyage. FLC, D3-02-72.

30 “...nous avons constitué, sous la direction de Mr. André Wogenscky, un groupe d'étude qui constitue en France le premier noyau du Groupe CIAM-Jeunes. Le premier sujet proposé par Mr. Wogenscky étant l' «Habitat», nous serions très heureux de pouvoir donner, dès le Congrès de Stockholm, une première relation de nos travaux d'études" dans la lettre de Claude Parent et Ionel Schein à Siegfried Giedion du 24 mars 1952. GTA, 42-SG-40-506.

31 "Monsieur Wogenscky a donné une première impulsion à cette idée [CIAM Jeunes], en réunissant dans une série de causeries sur l'Habitat, un premier groupe d'étudiants" dans Schein, Ionel; Parent, Claude: Présences, 23 juin 1952. GTA.

${ }^{32}$ Cfr. Bonillo, Jean-Lucien; Massu, Claude; Pinson, Daniel (édité par): La modernité critique, autour du CIAM 9 d'Aix-enProvence - 1953. Marseille: Imbernon, 2006.

${ }^{33}$ Schein, Ionel; Parent, Claude: Premières conclusions du Congrès. L’Architecture d’Aujourd'hui. №48, 1953, p. V. Parent n'ayant pas participé au Congrès, l'article a été écrit par Ionel Schein (voir le manuscrit de 3 pages au FRAC). L'article est présent en 2 copies à la FLC : D3-02-492/ D3-02-494 ; D3-02-500/ D3-02-504.

${ }^{34}$ Schein, Ionel: Peuple et architecture populaire. Architecture. $N^{\circ} 38,1961$, pp. 672-678

${ }^{35}$ Schein, Ionel; Parent, Claude: Essai 1953.

${ }^{36}$ Schein, Ionel: CIAM 9, manuscrit, s.d. FRAC.
} 


\section{Où diable pêchez-vous votre jargon, vous autres, les jeunes? Formation d'un vocabulaire d'architecture à réaction sociale}

La correspondance entre Schein et Le Corbusier est ponctuée d'envoi d'articles, manifestes, idées, à partir du déjà cité compte-rendu du CIAM IX. Vers la fin des années Cinquante, Schein joue le rôle de correspondant de Paris pour plusieurs revues italiennes, suisses et allemandes d'architecture. Cette affectation lui est attribuée en raison de sa connaissance des langues étrangères et de sa renommée internationale après le succès de la Maison en Plastique en 1956. Sur les pages de la zevienne "L'architettura. Cronache e storia" il publie une série d'articles consacrés à l'architecture moderne dans la capitale française. La troisième Lettre de Paris ${ }^{37}$ est dédiée à Georges Candilis et Le Corbusier écrit à Schein: "J'avais pris connaissance de votre article dans la revue "Architettura" que je reçois. Je suis très content de voir que les anciens de la rue de Sèvres font de bonnes choses et que vous appréciez les travaux de Candilis et de Woods. Merci de la sympathie que vous avez pour mon travail ; j'ai déjà eu l'occasion de l'apprécier" ${ }^{\prime \prime 3}$. C'est Le Corbusier lui-même à souligner une différence en se référant à Candilis et Woods comme à des anciens dans la lettre adressée à Schein. Mais cette lettre révèle aussi une affinité, un lien plus ou moins évident, entre les différentes générations d'architectes qui se forment à l'atelier Le Corbusier, qu'il s'agit d' "enfants de Le Corbusier" ${ }^{\prime \prime 39}$, comme Parent et Schein, ou bien d' "anciens de la rue de Sèvres" ${ }^{\prime \prime 0}$.

Correspondant pour "Baukunst+Werkform", Ionel Schein est chargé de la rédaction d'un numéro monographique de la revue sur l'architecture contemporaine à Paris. La curatelle du projet est le moteur pour la préparation d'une exposition itinérante ${ }^{41}$ inaugurée à Hannover à l'occasion de la publication, en décembre $1959^{42}$ (Figures 3 et 4 ).
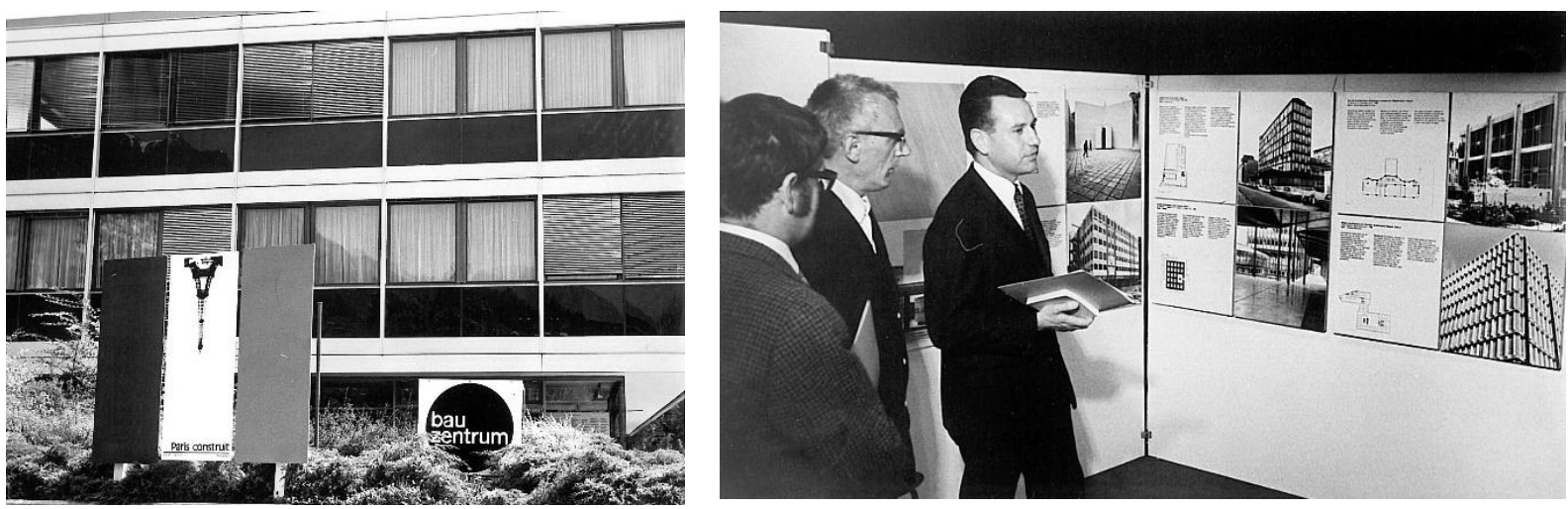

3. - 4. Ionel Schein (édité par), Exposition itinérante Paris baut/Paris construit, Hannover, 1959. Fonds Schein, FRAC Centre, Orléans.

\footnotetext{
${ }^{37}$ Schein, Ionel: Lettre de Paris: l'architecte Georges Candilis (3). L’architettura. Cronache e storia. №33, juillet 1958, pp. $167-175$.

${ }^{38}$ Lettre de Le Corbusier à Ionel Schein du 28 juillet 1958, FLC R3-03-61.

39 "Enfants de Le Corbusier, nous les fumes, Ionel Schein et moi..." dans Parent, Claude 1975, p. 28.

40 "Je suis très content de voir que les anciens de la rue de Sèvres font de bonnes choses et que vous appréciez les travaux de Candilis et de Woods". Lettre de Le Corbusier à Ionel Schein du 28 juillet 1958, FLC R3-03-61.

${ }^{41}$ Quelques étapes de l'exposition Paris baut/Paris construit présentée à Hannover, Allemagne (11 décembre 1959-11 janvier 1960), Musée des Arts Décoratifs, Paris (1960), Libano et Cipre (1965), Pavillon Baltard, Paris (1970), Kunstgewerbmuseum, Zurich (1972).

${ }^{42}$ Schein, Ionel: Paris baut. Baukunst+Werkform. Décembre 1959.
} 
L'exposition est indépendante d'aucun organisme, fondation culturelle ou parrainage français, afin de maintenir une position neutre dans la sélection et la présentation des projets. Les photographies sont réalisées par le suisse Thomas Cugini et par Ionel Schein, qui est l'auteur des textes.

La "sympathie"43 (dans le sens étymologique du terme) pour l'œuvre de Le Corbusier conduit Schein à présenter plusieurs bâtiments du maître dans son Paris baut, devenu deux ans plus tard une guide multilingue en format de poche au titre Paris construit (Figure 5).

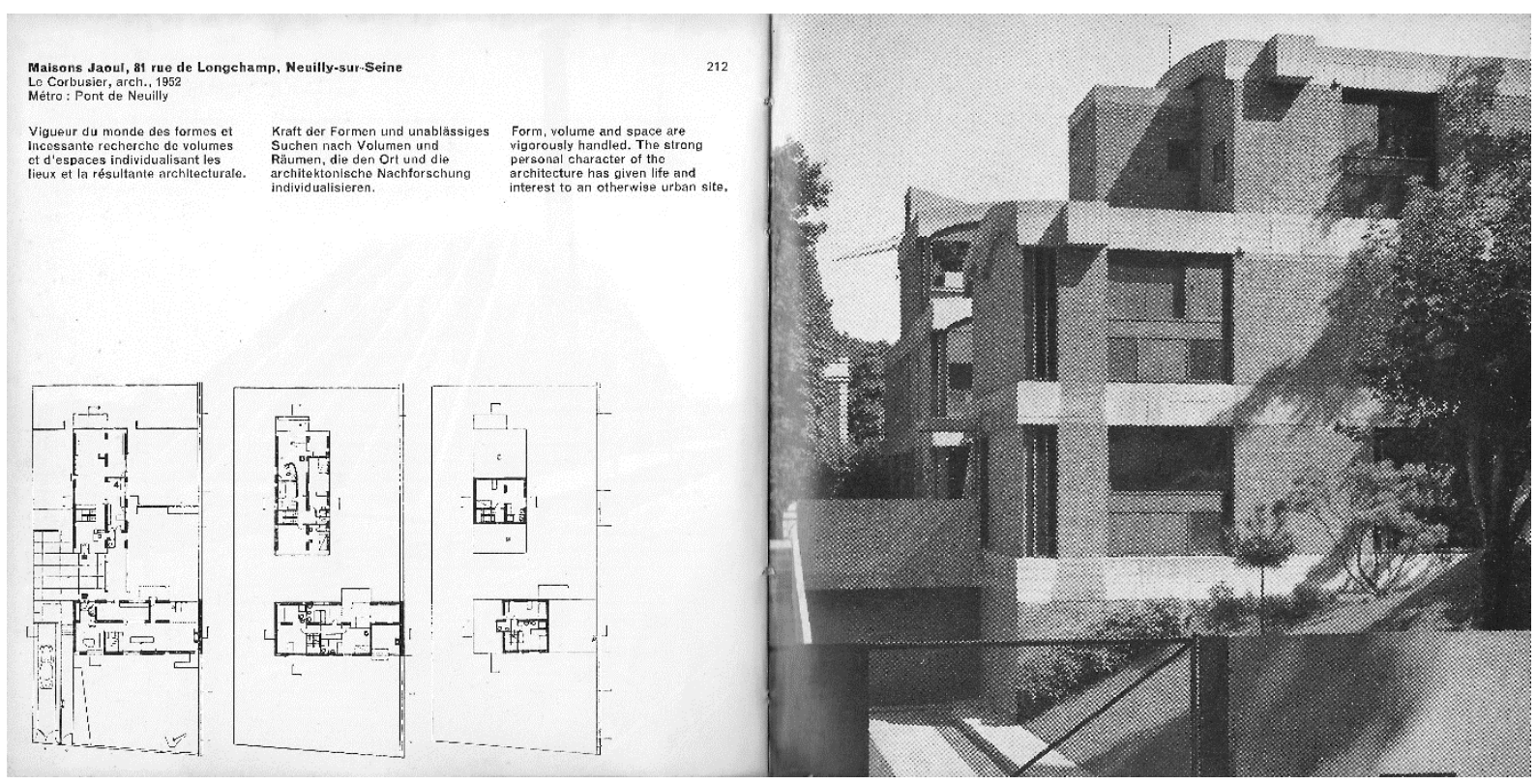

5. Ionel Schein, Paris construit, deux pages du guide dédiés aux Maisons Jaoul, photographiés par Ionel Schein. Dans Schein, Ionel: Paris construit. Guide de l'architecture contemporaine. Paris: Vincent Fréal et Cie, 1961, pp. 72-73; $1970\left(2^{\mathrm{e}}\right.$ ed. augmentée), pp. 212-213.

Schein demande à Le Corbusier les plans de la Maison du Brésil, que l'éditeur Girsberger est en train de redessiner pour l'Euvre complète ${ }^{44}$. Il reçoit les dessins le jour 7 novembre $1959^{45}$ : le plan publié dans Paris construit (première édition de $1961^{46}$ et deuxième augmentée de $1970^{47}$ ) est celui de l'CEuvre complète ${ }^{48}$, tandis que dans "Baukunst+Werkform" on trouve des dessins différents, probablement de la main de Schein.

Dans la revue, l'œuvre théorique de Le Corbusier prends une grande place, tandis que ce rôle est redimensionné dans le guide. L'introduction de "Baukunst+Werkform" est illustrée par un croquis du Plan Voisin ${ }^{49}$ et suivie par

\footnotetext{
${ }^{43}$ Lettre de Le Corbusier à Ionel Schein du 28 juillet 1958, FLC R3-03-61.

44 "Cher Monsieur, réponse à votre lettre du 1.9.1959, je regrette vivement mais je n'ai pas à disposition les documents graphiques que vous me réclamez. Les dessins sont faits au crayon; ils sont nombreux, successifs et rectificatifs. Je crois savoir que les Editions Ginsberger font une mise au net de ces dessins pour une publication. J'essaierai de me renseigner et de savoir si je pourrais obtenir pour vous une épreuve graphique utile" dans la lettre de Le Corbusier à Ionel Schein du 7 septembre 1959, FLC R3-03-65.

45 "Donné tout à Schein le 7 novembre 1959", note manuscrite sur la lettre de Ionel Schein à Le Corbusier du 14 septembre 1959, FLC R303-66.

${ }^{46}$ Schein, Ionel: Paris construit. Guide de l'architecture contemporaine. Paris: Vincent Fréal et Cie, 1961, p. 38.

${ }^{47}$ Schein, Ionel: Paris construit. Guide de l'architecture contemporaine, introduction par Max Querrien. Paris: Vincent Fréal et Cie, 1970 ( $2^{\mathrm{e}}$ ed. augmentée), p. 74.

${ }^{48}$ Le Corbusier; Boesinger, W. (édité par): Euvre complète 1957-65. Bâle: Birkhäuser, 2006, p. 192 (ed. or. Zürich: Éditions d'architecture, 1965).

${ }^{49}$ Republié dans Le Corbusier: Les Plans de Paris: 1956-1922. Paris: Éditions de Minuit, 1956, pp. 5, 137.
} 
l'essai Continuer! $!^{50}$, signé par Le Corbusier. Ce texte est accompagné par les dessins de la capitale française déjà publiés dans Précisions sur un état présent de l'architecture et de l'urbanisme ${ }^{51}$ et dans Les Plans de Paris : 1956-1922 $2^{52}$. La postface est aussi signée par Le Corbusier, qui propose une réflexion originale ${ }^{53}$ sur l'Unité de Nantes-Rézé, en soutenant la thèse de l'haute densité en architecture. Schein remercie chaleureusement Le Corbusier pour sa collaboration: "Cher Monsieur, je vous dois des remerciements sincères et frénétiques pour les documents que vous avez mis à ma disposition - et pour celui que vous m'avez donné. L'exposition "Paris Baut" sera présentée à Paris aussi et votre écriture, et vos idées et aussi quatre de vos réalisations (Pav. Suisse, Pav. Brésil, Rue Nungesser et Coli, Maisons Jaoul). Et je pense aussi ... à cet immense désir que j'ai (après avoir travaillé trois mois chez vous il y a six ans) de pouvoir parler avec vous, un soir, un matin, n'imported'architecture, donc de tout. Ne me renvoyez pas à vos livres; je les possède tous, et je les ai tous lu" ${ }^{\prime 54}$. Dès ce moment les contacts et les visites au Molitor sont plus fréquents et Schein invite Le Corbusier au vernissage de l'exposition Paris construit, qui rejoint finalement la capitale le 11 mars 1960 au Musée des Arts Décoratifs.

Les lettres de Schein sont souvent accompagnées par des articles publiés, mais aussi par des programmes inédits, des manifestes, des essais critiques. Le 25 juin 1960, Le Corbusier reçoit un texte de la part de Schein et il lui répond comme à un enfant: "J'ai lu avec vif intérêt votre papier : "Notes pour une attitude actuelle concernant l'Architecture». Vous avez parfaitement raison d'un but à l'autre. Vous êtes sur la piste depuis quelques années ; j’y suis depuis cinquante années" ${ }^{\prime 55}$. Le ton bourru ne décourage pas Schein, qui continue à envoyer au maître ses textes, comme Pour Paris du 1962. La réponse ne tarde pas, avec un post scriptum ironique : "Où diable pêchez-vous votre jargon, vous autres, les jeunes? Est-ce de la littérature de l'Est?" ${ }^{\prime 56}$.

L'indulgence qui accompagne le langage des jeunes suit également leurs gestes : pendant l'été 1960, Schein visite plusieurs architectures corbuséennes, y compris le pavillon caché de Roquebrune-Cap Martin et le chantier de l'Etoile de Mer. Les regards se croisent, mais la rencontre se fait en silence : Schein craint d'être chassé, tandis que Le Corbusier a peur que les gens découvrent et violent son retrait secret. Le même jour $1^{\mathrm{er}}$ septembre $1960^{57}$, les deux s'écrivent pour se rassurer réciproquement et se remercier pour ce silence complice.

$\mathrm{Au}$ contact de l'architecture corbuséenne (la Tourette surtout), la prose de Schein acquiert des tons lyriques: "Et d'où que l'on regarde, le couvent prend et garde l'aspect d'un tout - d'une sorte de miracle voulu, prévu, accompli. L'Homme de partout devrait, ici comme à Ronchamp, comme sur le toit de Marseille, comme à Nantes, comprendre que le beau est la vie ${ }^{\prime \prime 58}$. Le secret de Roquebrune renforce le lien et Le Corbusier participe à plusieurs évents organisés par Schein, comme la signature de son L'Atelier de la recherche patiente chez Vincent et Fréal. A cette occasion Le Corbusier rencontre les étudiants du cours tenu par Schein à l'École des Arts Décoratifs et c'est tout à fait l'occasion de boucler la boucle, par rapport à l'époque de la formation du

\footnotetext{
${ }^{50}$ Le Corbusier: Continuer!, dans Schein, Ionel: Paris baut, 1959, pp. 668-669.

${ }^{51}$ Le Corbusier: Précisions sur un état présent de l'architecture et de l'urbanisme. Paris: Éditions Crès, Collection de "L'Esprit Nouveau", 1930.

${ }^{52}$ Le Corbusier: Les Plans de Paris: 1956-1922. Paris: Éditions de Minuit, 1956, pp. 126-127.

53 "Das nebenstehende Blatt gab uns Le Corbusier als seinen Beitrag zu dem vorliegenden Heft und zur Ausstellung Paris Baut". Le Corbusier, Kleines Manifest zum Thema "Unité d'Habitation”, dans Schein, Ionel: Paris baut. 1959, pp. 706-707.

${ }^{54}$ Lettre de Ionel Schein à Le Corbusier du 3 décembre 1959, FLC R3-03-68.

${ }^{55}$ Lettre de Le Corbusier à Ionel Schein du 30 juin 1960, FLC R3-03-80. Cfr. Le Corbusier; Jenger, Jean (édité par): Choix de lettres. Bâle: Birkhäuser, 2002, pp. 453-454.

${ }^{56}$ Ibidem.

${ }^{57}$ Lettre de Le Corbusier à Ionel Schein du $1^{\text {er }}$ septembre 1960, FLC R3-03-81. Lettre d'Ionel Schein à Le Corbusier du $1^{\text {er }}$ septembre 1960, FLC R3-03-82.

${ }^{58}$ Lettre d'Ionel Schein à Le Corbusier du $1^{\text {er }}$ septembre 1960, FLC R3-03-82.
} 
jeune, quand le nom de ce «monsieur » était interdit. Pour les étudiants des cours de Schein, Le Corbusier écrit le texte L'urbanisme est l'expression de l'activité d'une époque. L'architecture est l'expression de l'esprit d'une époque ${ }^{59}$, destiné à être lu pendant la projection des diapositives de ses projets et dessins.

Lors que Le Corbusier est chargé du projet pour l'Hôpital de Venise, Schein lui envoie une note de félicitations avec le dessin de la place Saint Marc. Le Corbusier fait ses corrections au dessin (Figure 6), qu'il renvoie à Schein en photocopie, avec la lettre : "Cher Monsieur, merci de vos «Très sincères félicitations» du 16.11.1963. Je vous signale que la piazetta [sic] de Venise possède deux colonnes entre le Palais Ducal et l'autre Palais et qu'entre ces deux colonnes on aperçoit le Campanile et le fronton de l'île St Georges Majeur et qu'à Venise l'horizon est beaucoup plus bas que vous ne l'indiquez. Par ailleurs, les gondoles ne sont jamais mises à quai parallèlement mais transversalement et je vous signale, encore, que le Palais Ducal a un dessin de façade en marbre rose et blanc en losange et que les chapiteaux de la colonnade de ce Palais sont à la naissance des arcs. Bien cordialement à vous. Le Corbusier. P.J.: 1 photo-copie ${ }^{, 60}$.

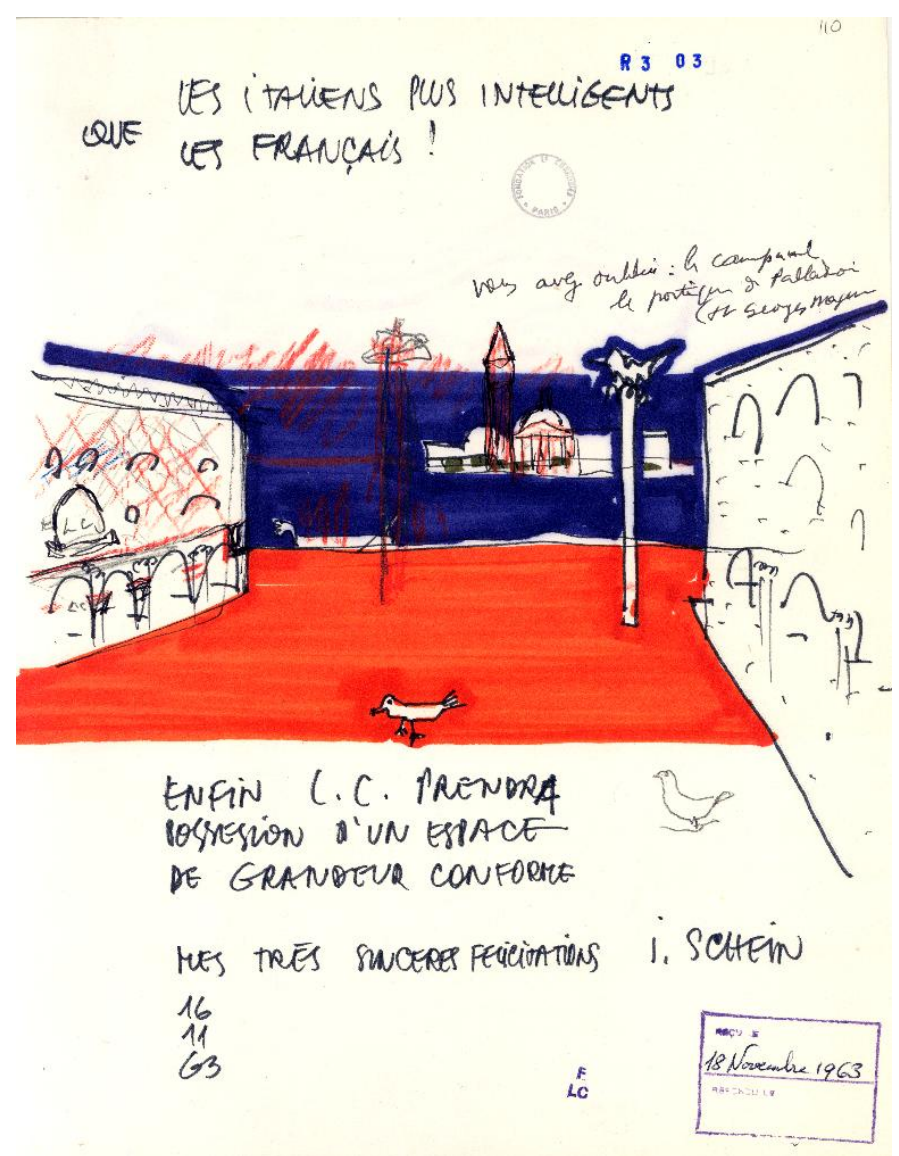

6. Note de félicitations de Ionel Schein à Le Corbusier du 16 novembre 1963. Corrections faites par Le Corbusier. Fondation Le Corbusier, Paris.

La publication d'une sélection de la correspondance corbuséenne Choix de lettres ${ }^{61}$, par Jean Jenger, reproduit cette lettre à Schein, mais l'éditeur coupe le texte après la signature, en oubliant le "par joint" et le dessin. La

\footnotetext{
${ }^{59}$ Lettre de Le Corbusier à Ionel Schein du 17 mai 1962, FLC R3-03-98. Cfr. Le Corbusier; Jenger, Jean (édité par): Choix de lettres. Bâle: Birkhäuser, 2002, pp. 478-479.

${ }^{60}$ Lettre de Le Corbusier à Ionel Schein du 2 décembre 1963, FLC R3-03-109-001. Dessin: FLC R3-03-109-002.

${ }^{61}$ Le Corbusier; Jenger, Jean (édité par): Choix de lettres. Bâle: Birkhäuser, 2002, p. 492.
} 
lettre devient ainsi un épanchement injustifiable et perd son caractère de divertissement intellectuel entre élève et enseignant, typique de leur correspondance. Le dessin de Venise est la synthèse parfaite de ce rapport, qui commence à l'école CIAM de 1952 et se termine dans la ville lagunaire, où les deux se rencontrent, probablement pour la dernière fois, en avril $1965^{62}$.

Schein a l'opportunité de connaitre, ou bien la capacité de faire ressortir, un Le Corbusier qui se plie volontiers à corriger les mots et les dessins de ses petits-enfants. Comme un grand-père bourru et imposant, il suit, avec une touche de fierté, leur pas sur le chemin qu'il a ouvert.

\section{Le cadavre rêvé Une suite grotesque d'honneurs posthumes}

Ionel Schein a dédié de nombreux écrits à la mémoire de Le Corbusier, publiés juste après sa mort en 1965 ou bien quelques années plus $\operatorname{tard}^{63}$. Le premier obituaire, publié sur "Architecture 65" en septembre-octobre, présente un caractère plus passionné et est partagé en deux sections. Tout d'abord La Mort, description du rituel funèbre qui met en scène, "machiavéliquement, la "perte" du "génial" et du "grand" Le Corbusier"64 ; ensuite La Vie, célébration de l'héritage de celui qui "fut philosophe - et plus : bâtisseur de sociétés; car il est indéniable qu'avant toute chose Le Corbusier est un des grands "structurateurs sociaux" de son temps" ${ }^{\prime 65}$. Les célèbres portraits de Le Corbusier aux lunettes noires qui accompagnent l'article sont faits par Thomas Cugini, le photographe de Paris construit, sur indication de Schein (Figure 7). Le croquis Venise: la cité à l'échelle humaine (Figure 8) a probablement été donné à Schein pendant leur rencontre dans la ville lagunaire en 1964, et constitue une réponse graphique au dessin de la Place Saint Marc que les deux s'étaient renvoyé l'année auparavant (Figure 6).

\footnotetext{
62 «L-C a vu Schein à Venise (8-14 Avril 65)» note manuscrite sur la lettre d'Ionel Schein à Le Corbusier du 3 Mars 1965, FLC R3-03-119.

${ }^{63}$ Voir, par exemple, les nombreux obituaires : Schein, Ionel: Le Corbusier, bâtisseur de sociétés. Architecture $65 . \mathrm{N}^{\circ} 66$, septembre-octobre 1965; Id., Le Corbusier, bâtisseur de sociétés. Le Monde. 1 février 1967; Id., Le Corbusier pas mort. Un bâtisseur de sociétés. Le quotidien de Paris. 3 septembre 1975; Id., Ils étaient tous là... Architecture. Novembre 1981.

${ }^{64}$ Schein, Ionel: Le Corbusier, bâtisseur de sociétés. Architecture 65. №66, septembre-octobre 1965

${ }^{65}$ Ibidem.
} 

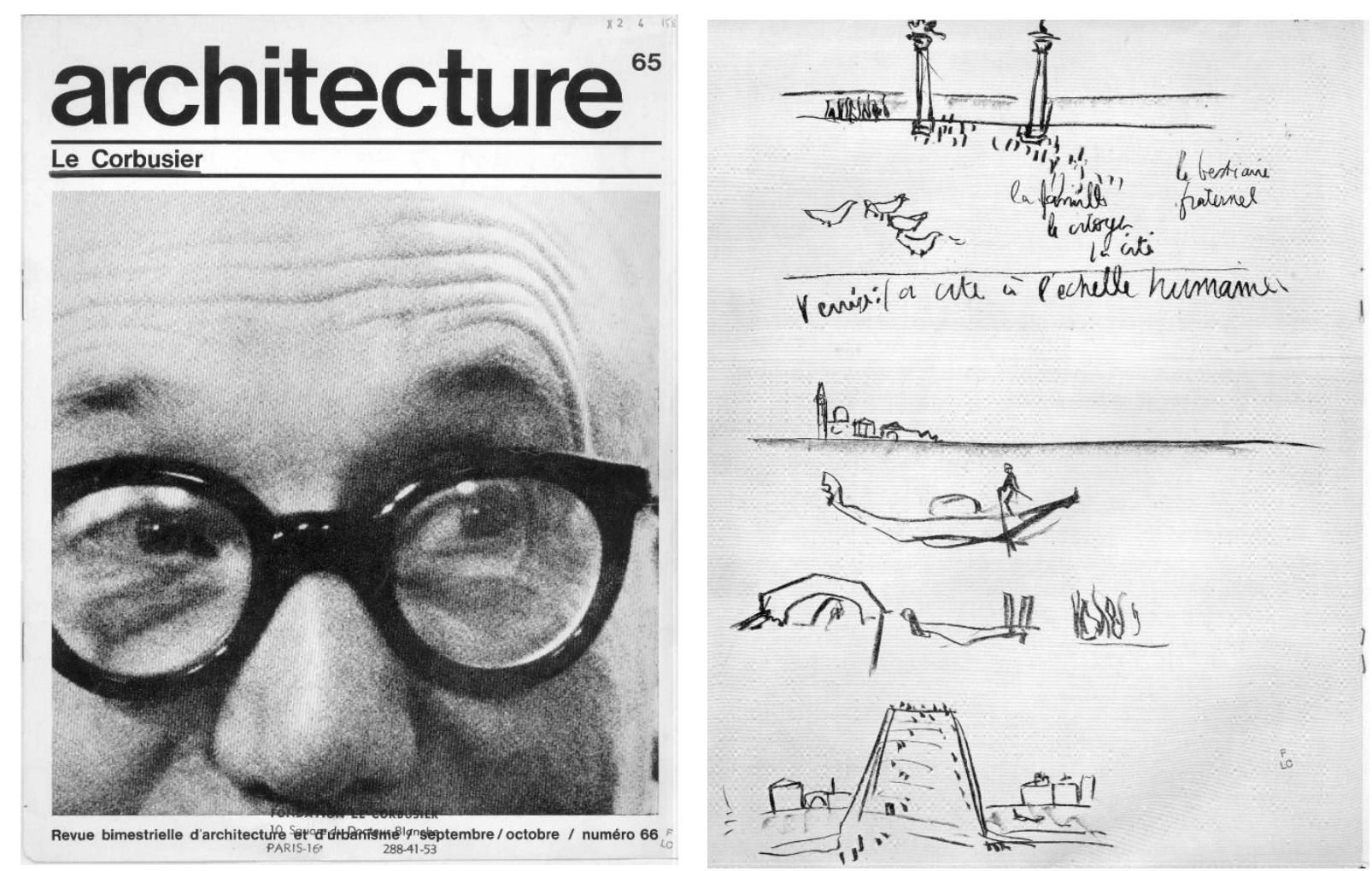

7. Le Corbusier, Couverture de l'édition monographique commémorative de la revue Architecture 65, septembre-octobre 1966. Portrait par Thomas Cugini.

8. Croquis de Le Corbusier Venise : la cité à l'échelle humaine, publié dans la même revue, Architecture 65, septembreoctobre 1966.

Les funérailles somptueuses réservées à Le Corbusier arrachent à Schein de considérations pleines de résignation: "... en France cartésienne, les hommes de valeur n'acquièrent leur grandeur aux yeux de ceux qui les ont humiliés durant leur vie, qu'après leur mort. Ce besoin de «disparition » est indispensable à ceux qui, à la tête des affaires du pays, ont recours aux alibis sécurisants: Le Corbusier était par son caractère intransigeant, sa vie austère et sa mort subite, pour cette besogne, le cadavre rêvén ${ }^{\prime \prime 66}$.

En conclusion on pourrait relire, cinquante ans après la mort de Le Corbusier, les mots que Schein a écrit dix ans après cette même date. Dans une époque actuelle de polémique intense, on oublie trop souvent que "L'actualité de Le Corbusier réside dans son esprit imaginatif et inventif, innovant; dans la poursuite incessante et sans faille d'un même combat farouche, celui pour l'architecture, dans l'absence de tout compromis. Dans l'histoire de l'architecture contemporaine sa place n'est pas encore prise ; il vit toujours ${ }^{\prime \prime 67}$.

\section{Bibliographie}

Berselli, Silvia: Ionel Schein. Dall'habitat evolutivo all'architecture populaire. Mendrisio: Mendrisio Academy Press - Cinisello Balsamo: Silvana Editoriale, 2015

Bonillo, Jean-Lucien; Massu, Claude; Pinson, Daniel (édité par): La modernité critique, autour du CIAM 9 d'Aix-en-Provence 1953. Marseille: Imbernon, 2006

\footnotetext{
${ }^{66}$ Ionel Schein, Le Corbusier pas mort. Un bâtisseur de sociétés. Le quotidien de Paris. 3 septembre 1975.

${ }^{67}$ Ionel Schein, Le Corbusier pas mort. Un bâtisseur de sociétés. Le quotidien de Paris. 3 septembre 1975.
} 
Epron, Jean-Pierre (édité par): Architecture: une anthologie. Tome 2: les architectes et le projet. Liège: Pierre Mardaga, 1992

Giedion, Siegfried: A Decade of New Architecture - Dix ans d'Architecture Contemporaine. Zurich: Girsberger, 1951

Le Corbusier: Précisions sur un état présent de l'architecture et de l'urbanisme. Paris: Éditions Crès, Collection de "L'Esprit Nouveau", 1930

Le Corbusier: Les Plans de Paris: 1956-1922. Paris: Éditions de Minuit, 1956

Le Corbusier; Boesinger, W. (édité par): Euvre complète 1957-65. Bâle: Birkhäuser, 2006 (éd. or. Zürich:

Éditions d'architecture, 1965)

Le Corbusier; Jenger, Jean (édité par): Choix de lettres. Bâle: Birkhäuser, 2002

Parent, Claude: Claude Parent Architecte. Paris: Laffont, 1975

Ragon, Michel: Monographie critique d'un architecte: Claude Parent. Paris: Dunod, 1982

Raimond, Anne-Marie: Un toit pour chaque français (1 et 2). Elle. №399, 27 juillet 1953, pp. 24-29; Elle. N $^{\circ}$ 400, 3 aout 1953, pp. 26-29

Schein, Ionel; Parent, Claude: Maison Française 1953. La Maison Française. N$^{\circ}$ 69, juillet 1953, pp. 3-14

Schein, Ionel; Parent, Claude: Premières conclusions du Congrès. L’Architecture d'Aujourd'hui. N 48,1953 , p. V

Schein, Ionel; Parent, Claude: Essai pour un habitat individuel évolutif et Habitation à Ville d'Avray.

L'Architecture d'Aujourd'hui. N 49, octobre 1953, pp. 4-5 et pp. 10-13

Schein, Ionel: Lettre de Paris: l'architecte Georges Candilis (3). L'architettura. Cronache e storia. N³3, juillet 1958, pp. 167-175

Schein, Ionel: Paris baut. Baukunst+Werkform. Décembre 1959

Schein, Ionel: Peuple et architecture populaire. Architecture. N³8, 1961, pp. 672-678

Schein, Ionel: Paris construit. Guide de l'architecture contemporaine. Paris : Vincent Fréal et Cie, 1961 (1e éd). 1970 (2e éd. augmentée)

Schein, Ionel: Le Corbusier, bâtisseur de sociétés. Architecture 65. №66, septembre-octobre 1965

Schein, Ionel: Le Corbusier, bâtisseur de sociétés. Le Monde. 1 février 1967

Schein, Ionel: Le Corbusier pas mort. Un bâtisseur de sociétés. Le quotidien de Paris. 3 septembre 1975

Schein, Ionel: Ils étaient tous là.... Architecture. Novembre 1981 